\title{
On Approximating Planar Metrics by Tree Metrics
}

\author{
Goran Konjevod* $_{\text {R. Ravi }}^{\dagger} \quad$ F. Sibel Salman ${ }^{\ddagger}$
}

\begin{abstract}
We connect the results of Bartal [4] on probabilistic approximation of metric spaces by tree metrics, and Klein, Plotkin and Rao [11] on decompositions of graphs without small $K_{s, s}$ minors (such as planar graphs) to show that metrics induced by such graphs can be probabilistically approximated by tree metrics with an $O(\log d)$ distortion, where $d$ is the diameter of the given graph. This improves upon Bartal's result that holds for general $n$-node metrics with a distortion of $O(\log n \log \log n)$. The main ingredient of our proof is that weak probabilistic partitions suffice for the construction of tree metrics with low distortion, in contrast to strong partitions used by Bartal. We also show that our result is the best possible by providing a lower bound of $\Omega(\log d)$ for the distortion of any probabilistic approximation of the square grid by tree metrics.
\end{abstract}

Keywords: Algorithms, Combinatorial problem, Metrics, Tree Metrics, Planar Graphs, Probabilistic Approximations

\section{Introduction}

\subsection{Metric approximations.}

Approximating a given metric by structurally simpler metrics has been an area of much research motivated by several different perspectives such as functional analysis [9], graph theory [7], distributed computation [14], approximation algorithms [12], and computational biology [1]. The general notion of approximation is to sandwich a simpler metric between the given metric and some multiple of the given metric. A popular simple metric used to approximate a given metric is an additive or tree metric, represented by distances arising from a tree containing the given points. The distances in the additive metric must dominate the distances in the graph but not be much greater. The distortion is the maximum ratio of any distance in the simpler metric to the original distance. However, even for the simple metric induced by an $n$-cycle, if we restrict ourselves to approximating this by tree metrics, the distortion must be at least $\Omega(n)[4,15]$.

To overcome this obstacle, Karp [10] introduced the notion of probabilistic approximations by simpler metrics, and showed how the cycle metric can be 2-approximated by a uniform distribution

${ }^{*}$ Dept. of Math. Sciences, Carnegie Mellon University, Pittsburgh, PA 15213-3890. Supported by an NSF CAREER grant CCR-9625297. Email: konjevod+@andrew.cmu.edu

${ }^{\dagger}$ GSIA, Carnegie Mellon University, Pittsburgh, PA 15213-3890. Supported by an NSF CAREER grant CCR9625297. Email: ravi@cmu.edu

‡GSIA, Carnegie Mellon University, Pittsburgh, PA 15213-3890. Email: fs2c+@andrew.cmu.edu 
on the path metrics obtained by deleting an edge of the cycle. The main idea is to give a polynomially computable probability distribution over a set of simpler metrics each of which dominates the given metric, and at the same time to keep the expected distortion for any edge small. Alon et al. [2] extended this result to show that every metric on $n$ points can be probabilistically approximated by tree metrics with distortion $2^{O(\sqrt{\log n \log \log n})}$.

Bartal [4] achieved a better distortion of $O\left(\log ^{2} n\right)$ and improved this bound to $O(\log n \log \log n)$ [5]. The technique underlying his results is an efficient computation of probabilistic (strong) partitions of the graph, where the induced graph on each cluster of the partition has a small diameter. The idea is to define a distribution over the set of strong partitions such that the probability of any edge being cut in a partition is small. This notion can be extended to define weak probabilistic partitions, where we no longer insist that the diameters of the induced graphs on the clusters be small, but only that any pair of nodes in a cluster are sufficiently close in the graph (i.e. we impose a bound on the "weak" diameter of the clusters, hence the name).

\subsection{Our results.}

In this paper, we first show that weak probabilistic partitions suffice to guarantee probabilistic approximations by $k$-HSTs (see definition in Section 2) with the same distortion of $O\left(\log ^{2} n\right)$ as in Bartal [4]. Our construction follows Bartal's, but results in $k$-HSTs in which the edge lengths along any root-leaf path decrease by exactly a factor of $k$.

Bartal gives a lower bound of $\Omega(\log n)$ respectively, for approximating the metric of any graph, leaving a small gap to close as the upper bound is $O(\log n \log \log n)$. We give an essentially tight result for graphs with unit-length edges which exclude a $K_{s, s}$ minor for some constant $s$. For example, planar graphs exclude $K_{3,3}$. We approximate the metrics of such graphs by tree metrics with $O(\log d)$ distortion, where $d$ is the diameter of the underlying graph. We obtain this improvement by randomizing a weak-diameter decomposition procedure due to Klein et al. [11] to get weak probabilistic partitions, and using the previous result to build the $k$-HSTs.

We then show that our result for graphs with small excluded minors is asymptotically the best possible by providing a lower bound of $\Omega(\log d)$ for the distortion of any probabilistic approximation of the square grid by tree metrics. Bartal's lower bound proof used sparse graphs of logarithmic girth which are known not to have small excluded minors due to the work of Robertson and Seymour. Our lower bound proof uses the easy direction of Yao's minimax theorem [17] and bounds the average distortion of any edge of the square grid in any tree-metric approximation by extending a result of Alon et al. [2].

Finally, we turn to applications of probabilistic approximation by tree metrics to network design problems $[3,6,16]$. In these applications, it is convenient if the tree metric is a spanning tree of the original metric. We give a simple "lifting" procedure to convert the approximating $k$-HSTs into spanning trees of the original metric with a factor of two increase in the expected distortion.

\section{Probabilistic approximation of metrics via weak partitions}

We use the following definitions from Bartal [4].

Definition 2.1. Given a metric space $M$ over a finite set $V$, let $d_{M}(x, y)$ denote the distance 
between two points $x, y \in V$. A family $\mathcal{F}$ of metric spaces over $V, \alpha$-probabilistically approximates $M$, if

$$
d_{M}(x, y) \leq d_{N}(x, y), \text { for all } N \in \mathcal{F}, x, y \in V,
$$

and there exists a probability distribution $\mathcal{D}$ on $\mathcal{F}$ such that

$$
\mathbf{E}_{\mathcal{D}}\left[d_{N}(x, y)\right] \leq \alpha d_{M}(x, y), \forall x, y \in V,
$$

where $N \in \mathcal{F}$ is chosen according to the distribution $\mathcal{D}$.

Definition 2.2. Let $G=(V, E, w)$ be a weighted connected graph. Consider a partition of the vertex set $V=\bigcup_{i} V_{i}$. The subgraph of $G$ induced by $V_{i}, G\left[V_{i}\right]$ is called a cluster of the partition. The strong diameter of the cluster is the diameter of $G\left[V_{i}\right]$, whereas the weak diameter of the cluster is the maximum distance in $G$ between two vertices of $V_{i}$.

Definition 2.3. An $\ell$-(weak) partition of $G$ is a partition of the vertex set $V=\bigcup_{i} V_{i}$ such that the strong (weak) diameter of $G\left[V_{i}\right]$ is at most $\ell$, for all $i$.

For a probability distribution $\mathcal{D}$ over all $\ell$-partitions of $G$, let $x_{e}$ denote the probability that edge $e$ crosses a boundary between some two clusters, i.e. its endpoints are in different clusters.

Definition 2.4. For any $0 \leq r \leq \operatorname{diamG}, \lambda, \rho \geq 0$, an $(r, \rho, \lambda)$-(weak) probabilistic partition of $G$ is a probability distribution $\mathcal{D}$ over the set of $(r \rho)$-(weak) partitions of $G$, such that

$$
x_{e} \leq \frac{\lambda w_{e}}{r}, \quad \text { for all } e \in E
$$

Definition 2.5. A $k$-hierarchically well-separated tree $(k-H S T)$ is a rooted weighted tree such that (1) the edge lengths from any vertex to all of its children are the same, and (2) the edge lengths along any root-leaf path are decreasing by a factor of at least $k$.

Bartal uses strong probabilistic partitions of a graph recursively to construct a $k$-HST that approximates the metric of the graph.

Theorem 2.6. [4] For all $r \geq 0$, if there is a polynomially computable $(r, \rho, \lambda)$-probabilistic partition of every subgraph of $G$, then $G$ can be $\alpha$-probabilistically approximated by a polynomially computable distribution on a set of $k$-HSTs each of diameter $O(\operatorname{diamG})$, for $\alpha=O\left(\rho \lambda k \log _{k}(\operatorname{diamG})\right)$.

We extend this theorem to weak probabilistic partitions.

Theorem 2.7. For all $r \geq 0$, if there is a polynomially computable $(r, \rho, \lambda)$-weak probabilistic partition of every subgraph of $G$, then $G$ can be $\alpha$-probabilistically approximated by a polynomially computable distribution on a set of $k$-HSTs each of diameter $O(\operatorname{diamG})$, for $\alpha=O\left(\rho \lambda k \log _{k}(\operatorname{diamG})\right)$.

Proof. We follow Bartal's construction but deviate from it in the recursive definition of the radius bounds on clusters to accommodate weak partitions. We first describe this construction of the family of $k$-HSTs that approximate distances in $G$ and then prove the claimed upper bound on the expected distortion. 
Let $r_{i}=\operatorname{diamG} /\left(\rho \mathrm{k}^{\mathrm{i}}\right)$ First, get an $\left(r_{1}, \rho, \lambda\right)$-weak probabilistic partition of $G$. Then, find an $\left(r_{2}, \rho, \lambda\right)$-weak probabilistic partition of each of the resulting subgraphs of $G$. At a generic iteration $i$, compute an $\left(r_{i}, \rho, \lambda\right)$-weak probabilistic partition of $G_{i}$. Let $C_{i+1}^{1}, \ldots, C_{i+1}^{s}$ be the resulting clusters. Recursively construct the $k$-HST's for each of these clusters by setting $G_{i+1}=C_{i+1}^{j}, j=1, \ldots, s$. Let the corresponding trees be $T_{i+1}^{j}$ with roots $q_{i+1}^{j}, j=1, \ldots, s$. We construct $T_{i}$ by adding the node $q_{i}$ and connecting it to each of $q_{i+1}^{j}$ by an edge of length $r_{i} \rho k / 2=\operatorname{diamG} /\left(2 \mathrm{k}^{\mathrm{i}-1}\right)$. We stop when $G_{i}$ consists of a single vertex, thus the leaves of the tree are the vertices of $G$. Let $T_{1}=T$. Note that $T$ is a $k$-HST with the additional property that all the edge lengths at a level are equal, and it has depth at most $\log _{k}(\operatorname{diamG})$.

First we show by induction that the distance in $T$ between any pair of nodes in $V(G)$ is at least the distance in $G$. The claim holds trivially when $G_{i}$ has a single node. Assume $d_{T_{i+1}}(u, v) \geq$ $d_{G}(u, v)$. If $u$ and $v$ are both in cluster $C_{i+1}^{j}$, then $d_{T_{i}}(u, v)=d_{T_{i+1}^{j}}(u, v) \geq d_{G}(u, v)$. Otherwise, $d_{T_{i}}(u, v) \geq \operatorname{diamG} / \mathrm{k}^{\mathrm{i}-1}=\rho \mathrm{r}_{\mathrm{i}} \mathrm{k}$, and the weak diameter of the cluster at the $i$-th level containing both $u$ and $v$ is at most $\rho r_{i-1}=\rho r_{i} k$, hence $d_{T_{i}}(u, v) \geq d_{G}(u, v)$.

We will now show that the expected distortion of distances in $T$ is $O\left(\lambda \rho k \log _{k}(\operatorname{diamG})\right)$. The main deviation from Bartal's proof is that we always relate distances in $T$ to those in the original graph, as our probabilistic partitions are weak.

First, we relate the diameter of $T_{i}$ to that of $G$. Let $T_{i}$ denote a subtree of $T$ whose root is a vertex at the $i$-th level of $T$ and let $\gamma\left(T_{i}\right)$ denote the maximal length of a root-leaf path in $T_{i}$. We prove by induction that $\gamma\left(T_{i}\right) \leq 1 / 2(1+1 /(k-1)) \operatorname{diamG} / \mathrm{k}^{\mathrm{i}-1}$. Assume the claim holds for $i+1$. Then,

$$
\gamma\left(T_{i}\right)=\max _{j} \gamma\left(T_{i+1}^{j}\right)+\frac{1}{2} \frac{\operatorname{diamG}}{\mathrm{k}^{\mathrm{i}-1}} \leq \frac{1}{2}\left(1+\frac{1}{k-1}\right) \frac{\operatorname{diamG}}{\mathrm{k}^{\mathrm{i}}}+\frac{1}{2} \frac{\operatorname{diamG}}{\mathrm{k}^{\mathrm{i}-1}} \frac{1}{2} \frac{\operatorname{diamG}}{\mathrm{k}^{\mathrm{i}-1}}\left(1+\frac{1}{k-1}\right) .
$$

Hence,

$$
\operatorname{diam}_{\mathrm{i}} \leq\left(1+\frac{1}{\mathrm{k}-1}\right) \frac{\operatorname{diamG}}{\mathrm{k}^{\mathrm{i}-1}} .
$$

Bartal's proof of the upper bound on the expected distance in $T$ carries over almost directly. The proof shows by induction on $i$ that for some $h \leq \log _{k}(\operatorname{diamG})$,

$$
\mathbf{E}\left[d_{T_{i}}(u, v)\right] \leq \lambda \rho k\left(1+\frac{1}{k-1}\right)(h-i) d_{G_{i}}(u, v) .
$$

Assume the claim holds for $i+1$. Let $A_{i}$ be the event that a shortest $u-v$ path is within some cluster $C_{i+1}^{j}$ produced at iteration $i$. Then,

$$
\begin{aligned}
\mathbf{E}\left[d_{T_{i}}(u, v) \mid A_{i}\right]=\mathbf{E}\left[d_{T_{i+1}^{j}}(u, v)\right] & \leq \lambda \rho k\left(1+\frac{1}{k-1}\right)(h-i-1) d_{C_{i+1}^{j}}(u, v) \\
& =\lambda \rho k\left(1+\frac{1}{k-1}\right)(h-i-1) d_{G_{i}}(u, v) .
\end{aligned}
$$


Let $x_{i}(u, v)$ be the probability that some shortest $u-v$ path crosses between some clusters produced at iteration $i$. Then,

$$
\begin{aligned}
& \mathbf{E}\left[d_{T_{i}}(u, v)\right] \leq x_{i}(u, v) \operatorname{diamT}_{\mathrm{i}}+\left(1-\mathrm{x}_{\mathrm{i}}(\mathrm{u}, \mathrm{v})\right) \mathbf{E}\left[\mathrm{d}_{\mathrm{T}_{\mathrm{i}}}(\mathrm{u}, \mathrm{v}) \mid \mathrm{A}_{\mathrm{i}}\right] \\
& \leq \lambda \frac{d_{G_{i}}(u, v)}{r_{i}} \operatorname{diamT}_{\mathrm{i}}+\mathbf{E}\left[\mathrm{d}_{\mathrm{T}_{\mathrm{i}}}(\mathrm{u}, \mathrm{v}) \mid \mathrm{A}_{\mathrm{i}}\right] \\
& \leq \lambda \frac{d_{G_{i}}(u, v)}{\operatorname{diamG}} \rho k^{i}\left(1+\frac{1}{k-1}\right) \frac{\operatorname{diamG}}{\mathrm{k}^{\mathrm{i}-1}}+\lambda \rho k\left(1+\frac{1}{k-1}\right)(h-i-1) d_{G_{i}}(u, v) \\
& =\lambda \rho k\left(1+\frac{1}{k-1}\right) d_{G_{i}}(u, v)+\lambda \rho k\left(1+\frac{1}{k-1}\right)(h-i-1) d_{G_{i}}(u, v) \\
& =\lambda \rho k\left(1+\frac{1}{k-1}\right)(h-i) d_{G_{i}}(u, v) \text {. }
\end{aligned}
$$

The first inequality follows since in a $\left(r_{i}, \rho, \lambda\right)$-weak probabilistic partition, $x_{i}(u, v) \leq \sum_{e \in P} x_{e}$, and thus $x_{e} \leq \lambda w_{e} / r_{i}$ implies $x_{i}(u, v) \leq \lambda \cdot d_{G_{i}}(u, v) / r_{i}$. The second inequality uses (1), the definition of $r_{i}$ and (2).

Finally, since $h \leq \log _{k}(\operatorname{diamG}), \mathbf{E}\left[d_{T}(u, v)\right] \leq \lambda \rho k\left(1+\frac{1}{k-1}\right) \log _{k}(\operatorname{diamG}) \mathrm{d}_{\mathrm{G}}(\mathrm{u}, \mathrm{v})$.

Remark: Bartal (personal communication, 1997) has pointed out that the diamG bound in the above proof can be replaced by $n$ by using the idea of $\frac{1}{n}$-forcing weak partitions as in [4].

\section{Probabilistic partition of graphs without $K_{s, s}$ minors}

In this section we randomize the decomposition of Klein et al. [11] to show that given a graph $G$ and parameters $\delta$ and $s$, either $G$ has a $K_{s, s}$ minor, or there is a $\left(\delta, O\left(s^{2}\right), s\right)$-weak probabilistic partition of any subgraph of $G$. This result together with Theorem 2.7 yields a family of $k$-HSTs that $O\left(s^{3} k \log _{k}\right.$ diamG)- probabilistically approximates the metric of $G$.

Motivated by applications to multicommodity flow problems, Klein et al. [11] prove the following:

Theorem 3.1. A graph $G$ without a $K_{s, s}$ minor can be decomposed into subgraphs of weak diameter $4 \delta s^{2}$ by removing a constant $(s / \delta$ ) fraction of edges, where $s<\delta<\operatorname{diamG}$.

We now describe the decomposition procedure. Let a parameter $\delta$ be given. Find a breadth-first search tree of $G$ starting from any vertex as the root. If the depth of the tree is less than $\delta$, stop. Otherwise pick some number $\eta \in\{1,2, \ldots, \delta\}$, and divide $V$ into clusters of vertices whose distance from the root is between $\eta+i \delta$ and $\eta+(i+1) \delta-1$, for $i=0,1, \ldots$ by deleting edges that cross the clusters. Recursively apply the same procedure to each cluster. The depth of the recursion is $s$, if $G$ has no $K_{s, s}$ minor (thus, $s=3$ for a planar graph). In [11], at each level of recursion, $\eta$ is picked so as to minimize the number of edges removed.

The key observation is that the guarantee on the weak diameter bound is independent of the choice of the root node or the choice of $\eta$ at any stage of recursion. We simply pick the root vertex and $\eta$ uniformly at random from the set of all available vertices and $\{1, \ldots, \delta\}$, respectively, at every level of the recursion. 
Theorem 3.2. If a graph $G$ doesn't contain a $K_{s, s}$ minor, then the decomposition procedure outputs $a\left(\delta, 4 s^{2}, s\right)$-weak probabilistic partition of $G$.

Proof. In [11] it is shown that the weak diameter of the clusters produced is at most $4 \delta s^{2}$. Therefore, each of the partitions that may result as a consequence of particular choices of the cutting parameter $\eta$ is a $4 \delta s^{2}$-weak partition of $G$. Since $\eta$ and the root are chosen uniformly at random, the probability that a given edge will be deleted is at most $1 / \delta$ at every level of the recursion. Since the recursion terminates after at most $s$ levels (see [11]), the union bound gives $x_{e} \leq w_{e} s / \delta$.

The following result easily follows from Theorems 2.7 and 3.2.

Corollary 3.3. Let $G$ be a weighted graph without a $K_{s, s}$ minor. Then $G$ can be $\alpha$-probabilistically approximated by a polynomially computable distribution on a set of $k$-HSTs, for $\alpha=O\left(s^{3} k \log _{k}(\operatorname{diamG})\right)$.

\section{Lower bounds}

In this section we show that the metric of a 2-dimensional $n \times n$ grid, which we denote by $G(n, 2)$, cannot be $\alpha$-probabilistically approximated by a tree for any $\alpha=o(\log n)$. The result is an extension of a theorem of Alon, Karp, Peleg and West [2].

An often used method of proving lower bounds for (either the running time or the performance guarantee of) randomized algorithms is that of Yao [17, 13], based on the minimax principle of linear programming. Consider a randomized algorithm as a distribution $p$ on some set of deterministic algorithms $A \in \mathcal{A}$. If $q$ is any distribution on the set of inputs $I \in \mathcal{I}$, then

$$
\min _{A \in \mathcal{A}} \mathbf{E}_{q}[\operatorname{cost}(I, A)] \leq \mathbf{E}_{p}\left[\max _{I \in \mathcal{I}} \operatorname{cost}(I, A)\right]
$$

Definition 4.1. Let $T$ be a spanning tree of the graph $G$. The distortion of an edge e with respect to $T$ is the length of the unique path in $T$ between the endpoints of e. We denote this by $\operatorname{dist}_{\mathrm{T}}(\mathrm{e})$.

Alon et al. [2] prove the following lower bound on the expected distortion.

Theorem 4.2. Let $T$ be a spanning tree of $G(n, 2)$. If $q$ is the uniform distribution on the edges of $G(n, 2)$, then

$$
\mathbf{E}_{q}\left[\operatorname{dist}_{\mathrm{T}}(\mathrm{e})\right] \geq \frac{1}{2048} \ln \mathrm{n}-\mathrm{O}(1)
$$

Note that from (4) it follows that any lower bound for expected distortion of an edge (like the one in Theorem 4.2) immediately translates to the same lower bound for probabilistic approximation by spanning trees. Thus we only need to show that the proof from [2] can be extended to the more general notion of probabilistic approximation by trees that are not necessarily subgraphs of the original graph.

First of all, we may assume that all the grid vertices are the leaves of the tree we consider (otherwise, add edges of length zero and make the grid vertices leaves). Further, we can reduce the maximum degree of the tree to 4 by splitting any vertex of degree greater than 4 into two vertices (dividing the neighbors between the two vertices) and connecting them by edges of length 0 . 
Definition 4.3. We call a tree $T$ a super-spanning tree of the grid $G=G(n, 2)$, if $(\mathbf{1}) d_{T}(x, y) \geq$ $d_{G}(x, y) \forall x, y \in V(G)$, (2) all vertices of $G$ are leaves of $T$, and (3) all vertices of $T$ have degree at most 4 .

Note that a super-spanning tree of a grid need not be a spanning tree. However, the only reason a spanning tree might not be a super-spanning tree is requirement (2) which is easily enforced by adding edges of cost 0 . Thus it will be sufficient to generalize Theorem 4.2 to super-spanning trees.

Lemma 4.4. [2] If $A$ is a set of at least $\alpha^{2}$ vertices of $G(n, 2)$, and $|A| \leq n^{2} / 2$, then there are at least $\alpha$ rows or $\alpha$ columns that $A$ intersects but does not fill.

The next two lemmata are similar to the ones used in [2].

Lemma 4.5. (variant of Lemma 6.4 of [2]) Let $T^{\prime}$ be a subtree of a super-spanning tree of $G(n, 2)$, and $A$ be the set of leaves of $T^{\prime}$. If $A$ contains at least $\alpha^{2}$ vertices (of $G(n, 2)$ ), where $|A| \leq n^{2} / 2$, and $B$ is a set of at most four vertices in $T^{\prime}$, then there are at least $\alpha / 2$ vertices in $A$ that have neighbors outside $A$ and have distance at least $\alpha / 16$ from each vertex of $B$.

Proof. By the previous lemma there is a set $S$ of at least $\alpha$ vertices in $A$ that are in distinct rows (or columns), and have neighbors outside $A$ (hence $T^{\prime}$, as well). Because distances in $T$ overestimate distances in $G$, any vertex of $B$ can be within $\alpha / 16$ of at most $\alpha / 8$ vertices of $S$ in $T^{\prime}$. So, at least $\alpha / 2$ vertices of $S$ are more than $\alpha / 16$ away from any vertex of $B$.

Lemma 4.6. (variant of Lemma 6.5 of [2]) If $T$ is a super-spanning tree of $G(n, 2)$ and $1 \leq \alpha \leq$ $n / 16$, then there are at least $n^{2} /(64 \alpha)$ edges e such that $\operatorname{dist}_{\mathrm{T}}(\mathrm{e}) \geq \alpha / 16$.

Proof. Since the maximum degree of $T$ is at most 4, there is an edge whose removal splits $T$ into two components that both have at least $\left(n^{2}-1\right) / 4 \geq n^{2} / 8$ leaves. Starting with $T$, we repeatedly delete an edge from the biggest component, so that the graph is split as evenly as possible. We delete $\left\lceil n^{2} /\left(8 \alpha^{2}\right)\right\rceil-1$ edges. Thus, the average number of leaves in the components is $8 \alpha^{2}$, and each of the $\left\lceil n^{2} /\left(8 \alpha^{2}\right)\right\rceil$ components has at least $\alpha^{2}$ leaves.

The average number of deleted edges incident with a component is less than 2, so at least half the components are incident with at most 4 deleted edges. Let $D$ be the set of such components and let $T^{\prime}$ be one of the components in $D$. Let $A$ be the set of leaves of $T^{\prime}$ and $B$ be the set of vertices in $T^{\prime}$ incident on the deleted edges. Then, by Lemma 4.5 there are at least $\alpha / 2$ leaves in $T^{\prime}$ that are at distance at least $\alpha / 16$ from each vertex of $B$, and that have neighbors outside $A$. The paths in $T$ from any of these $\alpha / 2$ vertices in $A$ to their neighbors outside $A$ must go through a vertex of $B$. Therefore, from the $n^{2} /\left(16 \alpha^{2}\right)$ components, we get a total of $n^{2} /(64 \alpha)$ edges whose distortion in $T$ is more than $\alpha / 16$.

Theorem 4.7. If $T$ is a super-spanning tree of $G=G(n, 2)$, and an edge is chosen uniformly at random from $E(G)$, then

$$
\mathbf{E}\left[\operatorname{dist}_{\mathrm{T}}(\mathrm{e})\right] \geq \frac{1}{2048} \ln \mathrm{n}-\mathrm{O}(1) .
$$

The proof uses Lemma 4.6 and sums over all $\alpha \in\{1, \ldots, n / 16\}$. 


\section{Obtaining a spanning tree of the original metric}

Many recent network design approximation algorithms use probabilistic approximations by tree metrics, e.g., the buy-at-bulk network design problems [3], the group Steiner tree problem [6], and the communication cost spanning tree problem [16]. The key idea is that the expected cost of an optimal solution in the tree metrics is at most $O\left(\log ^{2} n\right)$ times the optimum. Furthermore, it is easy to find the minimum or near-minimum cost network in a tree metric. To transform the solution in the tree metric to one in the original metric, it is convenient if the tree is a spanning tree of the original metric, so the solution edges form a subtree of this spanning tree. However, this is not the case in the $k$-HST construction due to the addition of new internal nodes.

We show that HSTs can be made spanning trees of the original metric (not necessarily of the original graph) while losing a factor of 2 in the expected distortion. This is similar to a lifting procedure of Jiang, Lawler and Wang [8]. The resulting spanning trees are not guaranteed to be hierarchically well-separated, but this is not a concern in the mentioned network design approximations.

Theorem 5.1. Any $k-H S T T^{\prime}$ resulting from weak or strong probabilistic partitions, can be replaced by a tree $T$ whose vertex set is $V(G)$, such that

$$
d_{G}(u, v) \leq d_{T}(u, v) \leq 2 d_{T^{\prime}}(u, v) .
$$

Proof. Pick some leaf $v$ of $T^{\prime}$ such that not all of its ancestors are vertices of $G$. Let $w$ be the first vertex on the path from the root of $T^{\prime}$ to $v$ that is not in $V(G)$. Shrink the path from $w$ to $v$ and identify $w$ with $v$ (now this becomes a vertex of $G$ ). Repeat this until all the vertices of the tree are vertices of the original graph $G$. Finally, multiply the lengths of all the edges by 2 .

Clearly, $d_{T}(u, v) \leq 2 d_{T^{\prime}}(u, v)$, as the distance could only have decreased in the shrinking process. To show the lower bound, let $u$ and $v$ be leaves of $T^{\prime}$ and $w$ be their least common ancestor. Let $l_{i}$ be the length of the edges from $w$ to its children. From the construction of the tree it follows that $d_{G}(u, v) \leq 2 l_{i}$. (using weak partitions, $2 l_{i}$ is an upper bound on the weak diameter of the subgraph represented by the node $w$ ). After the shrinking phase, $w$ will be replaced by a leaf of $T^{\prime}$. If this leaf is one of the vertices $u, v$, then $d_{T}(u, v)=2 l_{i} \geq d_{G}(u, v)$. If this is some other vertex of $G$ then $d_{T}(u, v) \geq 2 l_{i} \geq d_{G}(u, v)$.

Note that at the end of the shrinking algorithm, a spanning tree of the metric is obtained and we may replace the updated edge weights by the original weights in the metric.

\section{References}

[1] R. Agarwala, V. Bafna, M. Farach, B. Narayanan, M. Paterson, and M. Thorup. On the aprroximability of numerical taxonomy. In Proceedings of the 7thAnnual ACM-SIAM Symposium on Discrete Algorithms, pages 365-372, 1996.

[2] N. Alon, R. M. Karp, D. Peleg, and D. West. A graph-theoretic game and its applitacion to the $k$-server problem. SIAM J. Comput., 24(1):78-100, 1995. 
[3] B. Awerbuch and Y. Azar. Buy-at-bulk network design. In Proceedings of the 38thAnnual IEEE Symposium on Foundations of Computer Science, pages 542-547, 1997.

[4] Y. Bartal. Probabilistic approximation of metric spaces and its algorithmic applications. In Proceedings of the 37th Annual IEEE Symposium on Foundations of Computer Science, pages 184-193, October 1996.

[5] Y. Bartal. On approximating arbitrary metrics by tree metrics. In Proceedings of the 30thAnnual ACM Symposium on Theory of Computing, pages 161-168, 1998.

[6] N. Garg, G. Konjevod, and R. Ravi. A polylogarithmic approximation algorithm for the group steiner tree problem. In Proceedings of the 9thAnnual ACM-SIAM Symposium on Discrete Algorithms, pages 253-259, 1998.

[7] R. L. Graham and P. M. Winkler. On isometric embeddings of graphs. Trans. of the Amer. Math. Soc., 288:527-536, 1985.

[8] T. Jiang, L. Lawler, and L. Wang. Aligning sequences via an evolution: complexity and approximation. In Proceedings of the 26thAnnual ACM Symposium on Theory of Computing, pages 760-769, May 1994.

[9] W. B. Johnson and J. Lindenstrauss. Extensions of Lipshitz mappings into a Hilbert space. Contemporary Mathematics, 26:189-206, 1984.

[10] R. M. Karp. A 2k-competitive algorithm for the circle. manuscript, Aug. 1989.

[11] P. Klein, S. A. Plotkin, and S. Rao. Excluded minors, network decomposition and multicommodity flow. In Proceedings of the 25th Annual ACM Symposium on Theory of Computing, pages 682-690, May 1993.

[12] N. Linial, E. London, and Y. Rabinovich. The geometry of graphs and some of its algorithmic applications. Combinatorica, 15(2):215-245, 1995.

[13] R. Motwani and P. Raghavan. Randomized Algorithms. Cambridge University Press, 1995.

[14] D. Peleg and A. A. Schaeffer. Graph spanners. Journal of Graph Theory, 13(1):99-106, 1989.

[15] Y. Rabinovich and R. Raz. Lower bounds on the distortion of embedding finite metric spaces in graphs. Discrete and Computational Geometry, 19:79-94, 1995.

[16] B. Y. Wu, G. Lancia, V. Bafna, K.-M. Chao, R. Ravi, and C. Y. Tang. A polynomialtime approximation scheme for minimum routing cost spanning trees. In Proceedings of the 9thAnnual ACM-SIAM Symposium on Discrete Algorithms, pages 21-32, 1997.

[17] A. C.-C. Yao. Probabilistic computations: Towards a unified measure of complexity. In Proceedings of the 18thAnnual IEEE Symposium on Foundations of Computer Science, pages 222-227, 1977. 
This research was sponsored in part by National Science Foundation (NSF) grant no. CCR-0122581. 